\title{
Damage evaluation under complex fatigue loading conditions
}

\author{
L. Reis
}

IDMEC, Instituto Superior Técnico, Universidade de Lisboa, Av. Rovisco Pais, 1, 1049-001 Lisbon, Portugal luis.g.reis@tecnico.ulisboa.pt; bttp:/ /orcid.org/0000-0001-9848-9569

J. Caxias, H. Soares, P. R. Costa

Instituto Superior Técnico, Universidade de Lisboa, Av. Rovisco Pais, 1, 1049-001 Lisbon, Portugal

joaohcaxias@gmail.com,soares.bn@gmail.com,pedro.r.costa@ist.utl.pt

\section{Anes}

Instituto Superior de Engenharia de Lisboa, Instituto Politécnico de Lisboa, Rua Conselheiro Emídio Navarro, 1, 19559-007, Lisbon, Portugal

vanes@dem.isel.pt

\section{Freitas}

IDMEC, Instituto Superior Técnico, Universidade de Lisboa, Av. Rovisco Pais, 1, 1049-001 Lisbon, Portugal manuel.freitas@tecnico.ulisboa.pt

ABSTRACT. Fatigue damage and life assessment of multiaxial loadings is still an issue yet to resolve. Many methods have been proposed with promising agreements with the experimental results. However, the performance of such methods is, more than often, purely based on fatigue tests with simple loading conditions. In this work the stress scale factor (SSF) criterion and the virtual cycle counting (vcc) method are used to estimate fatigue life and damage accumulation with two damage accumulation rules. Fatigue tests were carried out with three different variable amplitude random loadings, applied to several specimens made from a 42CrMo4 high-strength steel. Fatigue crack plane measurements were compared with predictions from several critical plane models. The applied methods provided very acceptable results making the SSF package (SSF equivalent stress and virtual cycle counting method) a good method to estimate fatigue life and assessment of the damage accumulation in random fatigue loadings.

KEYwORDS. Multiaxial fatigue; Fatigue life; Damage accumulation; SSF; Critical plane models.

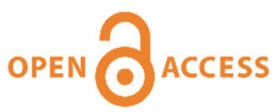

Citation: Reis, L., Caxias, J., Soares, H., Costa, P. R., Anes, V., de Freitas, M., Damage evaluation under complex fatigue loading conditions, Frattura ed Integrità Strutturale, 48 (2019) 318-331.

Received: 26.09.2018

Accepted: 28.11 .2018

Published: 01.04.2019

Copyright: (C) 2019 This is an open access article under the terms of the CC-BY 4.0, which permits unrestricted use, distribution, and reproduction in any medium, provided the original author and source are credited. 


\section{INTRODUCTION}

I n general, fatigue occurs in materials that are subjected to cyclic loadings. The consecutive loading and unloading cycles applied to the component, with amplitudes below the material's yield stress, will damage the component. Cycle by cycle the damage done to the material will accumulate, promoting fatigue crack's nucleation and growth, degradation of material properties and, ultimately, fracture [1]. Unlike static fracture, that occurs with visible plastic deformation, fatigue fracture will occur without warning, except for the cases where a crack is detected and its growth monitored before fracture. Depending on the role of a component in a mechanical structure, fatigue failure might have huge consequences, which can include life losses and great financial costs. One example, where mechanical fatigue failure is a major cause of incidents, is the aviation industry where, between 1927 and 1984, more than half of the documented incidents were due to fatigue failure [2].

The damage mechanism in uniaxial fatigue is well understood, and fatigue life is reasonably estimated. However, for multiaxial fatigue, which consists in the combination of stresses acting in two or more axes, is yet to be fully understood. Damage mechanisms depending on stress combinations, loading interaction effects, sequence effects, and cyclic plasticity are some of the many variables needed to be considered to assess fatigue damage in multiaxial loading conditions. Several studies were already conducted for the referenced parameters and many others related to multiaxial fatigue behavior [3-7]. Along the last decades, a great effort was made to correctly model the damage mechanism of multiaxial fatigue. However, most of the proposed criteria found in the literature has serious limitations to its usage. Most of the criteria proposed in the literature use parameters based in uniaxial testing data, or parameters that require extensive testing to determine them. Moreover, most of these criteria are evaluated using simple block loadings. However, when applied to complex nonproportional loadings, or random loadings, with variable stress amplitude, these models give poor or inconsistent results. When a new criterion is proposed, it should be validated with the most challenging task, which is to estimate fatigue life with non-proportional random loadings with variable stress amplitude. Many important authors in the multiaxial fatigue area of research have compared experimental results with many of the created models and with new and improved ones by themselves relating to the parameter of study [8-13].

This work aims to validate the Stress Scale Factor model (SSF) recently proposed and developed by Anes et al. [14] under those loading conditions.

\section{THEORETICAL BACKGROUND}

\section{SSF Criterion}

nes et al. [10] proposed a multiaxial fatigue criterion in a form of an equivalent shear stress. This criterion uses a new approach to calculate the stress scale factor. The stress scale factor (SSF) is often used in multiaxial fatigue criterions to convert an axial damage into shear's damage scale, or conversely. For example, in the von Mises criterion, the shear damage is updated into the axial damage scale, by multiplying the shear stress by a factor of $\sqrt{ } 3$. In previous works, the authors found that the loading path, the stress amplitude ratio and the stress level, all have influence in fatigue life. Therefore, using a constant SSF can lead to poor estimations. Using six different loading paths, where five were proportional and one was nonproportional, the authors obtained, through regression of fatigue data, a polynomial function, as shown in Eq. (1).

$$
\operatorname{ss} f\left(\lambda, \sigma_{a}\right)=a+b \cdot \sigma_{a}+c \cdot \sigma_{a}{ }^{2}+d \cdot \sigma_{a}{ }^{3}+f \cdot \lambda^{3}+g \cdot \lambda^{3}+h \cdot \lambda^{4}+i \cdot \lambda^{5}
$$

where $\mathrm{a}, \mathrm{b}, \mathrm{c}, \mathrm{d}, \mathrm{f}, \mathrm{g}$ and $\mathrm{h}$ are the polynomial fitting constants and are material dependent. Therefore, they need to be estimated with experimental fatigue data from the referenced six different loadings paths and stress levels. The fatigue results will give the S-N trendline for each applied loading path and thought them a corresponding SFF value can be calculated. It is using the different SFF values for each loading path that the polynomial constants of Eq. (1) can be calculated. $\lambda$ represents the stress amplitude ratio, and it is given by Eq. (2).

$$
\lambda=\tan ^{-1}\left(\frac{\tau_{a}}{\sigma_{a}}\right)
$$

where $\tau_{\mathrm{a}}$ and $\sigma_{\mathrm{a}}$ are the shear stress amplitude and the axial stress amplitude, respectively. This polynomial function can estimate the SSF for all stress amplitude ratios and stress levels for a given material. 
The SSF equivalent shear stress is given by Eq. (3).

$$
\tau_{e q v}=\tau_{a}+\operatorname{ssf}\left(\lambda, \sigma_{a}\right) \cdot \sigma_{a}
$$

To estimate fatigue life, the criterion uses the maximum equivalent shear stress as the damage parameter and uses the S-N trend line in pure shear loading conditions, as shown in Eq. (4).

$$
\left(\tau_{e q v}\right) \max =A\left(N_{f}\right)^{b}
$$

where $\mathrm{A}$ and $\mathrm{b}$ are the trend line parameters of the $\mathrm{S}-\mathrm{N}$ curve, obtained in pure shear loading conditions. For variable amplitude loading conditions, Anes et al. proposed a block extraction method. In this method, illustrated in Fig. 1, every time a $\tau_{\text {eqv }}$ peak greater than the previous peak is identified, a block is defined and a new block starts. This new maximum value of $\tau_{\text {eqv }}$ is the reference equivalent shear stress for the next loading block. The method is applied until all blocks have been identified.

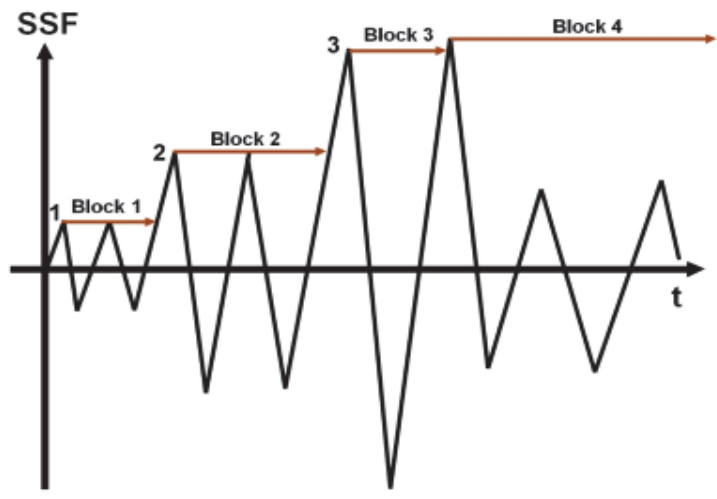

Figure 1: Block extraction method [15].

The number of cycles to failure, for each extracted block, is then calculated, as formulated in Eq. (5).

$$
\left(\tau_{\text {eqv }}\right)_{\max , \text { block }}=A\left(N_{f, \text { block }}\right)^{b}
$$

To calculate fatigue life, a cycle counting method must be used to count cycles in each extracted block. Then, the damage for each reversal can be determined by the ratio $n / N_{f}$ and summed with a damage accumulation rule.

\section{Critical plane models}

\section{Findley}

Findley [16] proposed a stress-based critical plane criterion that determines the critical plane as the one with the maximum damage parameter. Its formulation is given by Eq. (6).

$$
\min _{\theta}\left(\tau_{a}+k \sigma_{a, \max }\right)
$$

where, for each plane $\theta, \tau_{\mathrm{a}}$ is the shear stress amplitude, $\sigma_{\mathrm{a}, \max }$ is the maximum normal stress amplitude, and $\mathrm{k}$ is a stress scale factor, that must be determined experimentally. The k parameter varies between 0.2 and 0.3 for ductile materials.

\section{Brown-Miller}

After an extensive review Brown and Miller (B-M) concluded that both the octahedral shear strain and the maximum shear strain criteria fail to correctly describe the low-cycle fatigue behaviour. After combined tension and torsion fatigue testing, 
the authors proposed a strain-based criterion [17], with two strain parameters, depending on the type of fatigue crack nucleation and growth, given in Eq. (7) and Eq. (8).

For A type cracks:

$$
\left(\frac{\Delta \gamma}{g}\right)^{j}+\left(\frac{\varepsilon_{n}}{h}\right)^{i}=1
$$

For B type cracks:

$$
\frac{\Delta \gamma}{2}=\text { const }
$$

where $\Delta \gamma$ is the shear strain range and $\mathrm{g}, \mathrm{h}$ and $\mathrm{j}$ are material properties that must be determined experimentally. The same authors proposed a simplified formulation for A type cracks, which is given in Eq. (9).

$$
\frac{\Delta \gamma_{\max }}{2}+S \cdot \Delta \varepsilon_{n}
$$

where $\Delta \gamma_{\max }$ is the maximum shear strain range in a plane $\theta, \Delta \varepsilon_{\mathrm{n}}$ is the normal strain, in the plane of maximum shear strain, and $\mathrm{S}$ is the normal strain effect coefficient and is a material parameter.

\section{Fatemi-Socie}

Fatemi and Socie [18] (F-S) prosed a critical plane criterion that, compared to Brown-Miller's model, uses a normal strain parameter instead of the normal strain. The damage parameter is calculated using Eq. (10).

$$
\left[\frac{\Delta \gamma_{\max }}{2} \cdot\left(1+k \cdot \frac{\sigma_{n, \max }}{\sigma_{y}}\right)\right]_{\max _{\theta}}
$$

where $\sigma_{\mathrm{n}, \max }$ is the maximum normal stress in the plane of maximum shear strain amplitude, $\sigma_{\mathrm{y}}$ is the monotonic yield stress and $\mathrm{k}$ is a sensitivity parameter, that depends on the stress level.

When a loading has mean stress, the damage parameter is calculated as shown in Eq. (11).

$$
\max _{\theta}\left[\frac{\Delta \gamma_{\max }}{2} \cdot\left(1+k \cdot \frac{\sigma_{n}^{a}+\sigma_{n}^{m}}{\sigma_{y}}\right)\right]
$$

where $\sigma_{n}^{a}$ is the normal stress amplitude and $\sigma_{n}^{m}$ is the normal mean stress, both calculated in the plane with the maximum shear strain amplitude.

\section{Smith-Watson-Topper}

Smith et al. [19] developed a model, usually referred as the SWT model, to account the mean stress effect in uniaxial fatigue loading conditions.

Later on, Socie [20] used a critical plane approach to extend the usage of this model in multiaxial loading conditions. In these conditions, the damage parameter is given by Eq. (12).

$$
\max _{\theta}\left(\sigma_{n}\right) \cdot \frac{\Delta \varepsilon_{1}}{2}
$$

where $\sigma_{n}$ is the normal stress on a plane $\theta$ and $\Delta \varepsilon_{1}$ is the principal strain range in that plane. 


\section{Liu}

Liu's proposal [21] consists in an energy-based model with two different formulations, depending on fatigue failure's mode. For tensile failure mode the damage parameter, $\Delta W_{I}$ is given by Eq. (13), and by Eq. (14) for shear failure mode, $\Delta W_{I I}$.

$$
\Delta W_{I}=\max _{\theta}\left(\Delta \sigma_{n} \cdot \Delta \varepsilon_{n}\right)+(\Delta \tau \cdot \Delta \gamma)
$$

where $\Delta \sigma_{n}$ is the normal stress range, $\Delta \varepsilon_{n}$ is the normal strain range. $\Delta \tau$ and $\Delta \gamma$ are the shear stress range and the shear strain range, respectively.

$$
\Delta W_{I I}=\left(\Delta \sigma_{n} \cdot \Delta \varepsilon_{n}\right)+\max _{\theta}(\Delta \tau \cdot \Delta \gamma)
$$

This method uses a virtual strain energy (strain multiplied by stress) concept (VSE). The plane $\theta$ with the higher VSE will be the critical plane.

\section{Cycle counting}

As it was described, when a part or material sample is subjected with variable fatigue damage due to variable amplitude loading conditions, it is essential to design a model that can count the cycles in order to estimate the damage output in to the material. In this work three different counting methods were applied, the Rainflow, Virtual Cycle Counting and the Wang-Brown method. They are now briefly described.

\section{Rainflow counting method}

Matsuiski and Endo proposed cycle counting method based on the concept of a raindrop falling from a pagoda roof. The method identifies closed hysteresis loops, in a stress-strain plot, as cycles. A series of rules must be followed to extract cycles from a strain-time or stress-time history [22].

\section{Virtual cycle counting (vcc)}

Anes et al. [23] proposed a cycle counting method for variable amplitude multiaxial loadings, based on the SSF equivalent shear stress time evolution. In SSF equivalent shear stress history, the number of virtual cycles is given by adding the maximum absolute value of the peaks and valleys found between two zero stress points. This sum is then divided by the double of the maximum equivalent shear stress in the considered loading block. This method is formulated in Eq. (15).

$$
v c c_{\text {block }}=\frac{\sum a b s\left(\tau_{\text {eq }}\right)_{\text {peak }}+\sum a b s\left(\tau_{\text {eq }}\right)_{\text {valley }}}{2 \cdot\left(\tau_{\text {eq }}\right)_{\text {max }, \text { block }}}
$$

The formulation in Eq. (15) assumes that the block extraction method was applied. Otherwise, the denominator would be the maximum equivalent shear stress in the entire loading spectrum.

\section{Wang-Brown}

Wang and Brown [24] proposed a method to estimate fatigue life in variable amplitude loading conditions. In this method, it was proposed a new procedure to count cycles. Given a multiaxial strain history, the von Mises equivalent strain is calculated, as shown in Eq. (16).

$$
\varepsilon_{e q}=\frac{1}{\sqrt{2}\left(1+v^{\prime}\right)}\left\{\begin{array}{c}
{\left[\varepsilon_{x}-\varepsilon_{y}\right]^{2}+\left[\varepsilon_{y}-\varepsilon_{z}\right]^{2}+} \\
{\left[\varepsilon_{z}-\varepsilon_{x}\right]^{2}+} \\
\left(\frac{3}{2}\right)\left[\gamma_{x y}^{2}+\gamma_{y z}^{2}+\gamma_{z x}^{2}\right]
\end{array}\right\}^{\frac{1}{2}}
$$


Once the equivalent strain history is obtained, it is identified the maximum peak of the equivalent strain history, $\varepsilon_{i j}^{A}$, which will be the reference strain. All points prior to the time instant of $\varepsilon_{i j}^{A}, t_{A}$, are moved to the end of the history and the equivalent relative strain is then calculated, as shown in Eq. (17), resulting in a new strain history, where the first half-cycle goes from 0 to 2 .

$$
\varepsilon_{e q}(t)=\varepsilon_{e q}\left(\varepsilon_{i j}(t)-\varepsilon_{i j}^{A}\left(t_{A}\right)\right)
$$

After obtaining the relative strain history, it is performed the extraction of reversals. The extraction of a reversal starts at $t_{0}$, which once was $t_{A}$, and stops whenever a descending path is found. When this occurs the extraction of that reversal is resumed after an ascending path, with a higher relative strain, is found. This procedure goes on until the end of the relative strain history. Once a reversal is extracted, the equivalent shear strain data points, from this reversal, are removed from the original strain history, and the relative strain is again computed, followed by the extraction method. This procedure is conducted until there are no more reversals to extract.

Finally, the Wang-Brown's damage parameter is calculated for each reversal, as formulated in Eq. (18).

$$
\hat{\varepsilon} \equiv \frac{0.5\left(\Delta \gamma_{\max }\right)+S\left(\delta \varepsilon_{n}\right)}{1+v^{\prime}+\left(1-v^{\prime}\right) S}
$$

where $\Delta \gamma_{\max }$ is the maximum shear strain range, found in all the planes of projection, and $\delta \varepsilon_{n}$ is the normal strain excursion calculated between the time interval of $\Delta \gamma_{\max }$ and in the same plane of $\Delta \gamma_{\max } . v^{\prime}$ is the effective Poisson coefficient and $S$ is a material constant.

The number of reversals to failure, $2 N_{f}$, is then calculated for every reversal, as formulated in Eq. (19).

$$
\hat{\varepsilon}=\frac{\sigma_{f}^{\prime}-2 \cdot \sigma_{n, \text { mean }}}{E} \cdot\left(2 N_{f}\right)^{b}+\varepsilon_{f}^{\prime} \cdot\left(2 N_{f}\right)^{c}
$$

where $\sigma_{f}^{\prime}$ and $\varepsilon_{f}^{\prime}$ are the fatigue resistance coefficient and the fatigue ductility coefficient, respectively. $b$ and $c$ refer to the fatigue strength exponent and the fatigue ductility exponent.

The damage of a loading block is calculated using a linear damage accumulation rule, as shown in Eq. (20). Fatigue life, in blocks, is estimated as formulated in Eq. (21).

$$
\begin{aligned}
& D_{\text {block }}=\sum_{i=1}^{\# \text { of reversals }} \frac{1}{2 N_{f_{i}}} \\
& N_{\text {block }}=\frac{1}{D_{\text {block }}}
\end{aligned}
$$

\section{Damage accumulation rules}

In this work, two non-linear damage accumulation models were calculated for comparison, the Palmgren-Miner's rule and the Morrow's rule [25].

Palmgren proposed damage concept defined by the ratio between the number of cycles performed and the number of cycles to failure at a given load level. Using this concept, Miner proposed the so called Palmgren-Miner's Rule. This damage accumulation rule computes fatigue damage block by block and adds the damage linearly, as shown in Eq. (22).

$$
D=\sum_{i=1}^{k} \frac{n_{i}}{N_{i}}
$$

where $n_{i}$ and $N_{i}$ are the number of cycles in a block and the number of cycles to failure of the $i^{\text {th }}$ block, respectively, at a given stress level. $k$ is the number of stress levels present in the loading history. The rule states that the damage at failure moment is equal to 1 .

Morrow proposed a nonlinear damage accumulation rule that accounts for load interaction effects in variable amplitude loadings. This rule updates the Miner's damage by a parameter that is computed by the ratio between the maximum stress amplitude at the $i^{\text {th }}$ stress level and the maximum normal stress of the loading spectrum, as shown in Eq. (23). 


$$
D_{\text {block }}=\sum_{i=1}^{k} \frac{n_{i}}{N_{i}} \cdot\left(\frac{\sigma_{i}}{\sigma_{\max }}\right)^{d}
$$

where $d$ is the materials sensitivity to a variable amplitude loading history. This parameter is determined with cyclic properties of the material [26].

\section{EXPERIMENTAL PROCEDURE}

$\mathrm{F}$ ollowing up on previous works from other authors, the $42 \mathrm{CrMo} 4$ steel was selected for analysis. The low alloy steel DIN 42CrMo4 (AISI4140) is typically heat-treated quenching, and tempering. The chromium and molybdenum presence in the 42CrMo4 steel makes this alloy particularly suitable to get a variety of strength and ductility combinations, being widely used in automotive components such as crankshafts, front vehicle axles, steering components and hot forging components. In Tab. 1 it is shown the chemical composition of the steel and, in Tab. 2, it is summarized the monotonic and cyclic properties of the $42 \mathrm{CrMo} 4$ steel.

\begin{tabular}{cccccccccc}
\hline Element & $\mathrm{C}$ & $\mathrm{Si}$ & $\mathrm{Mn}$ & $\mathrm{P}$ & $\mathrm{S}$ & $\mathrm{Cr}$ & $\mathrm{Ni}$ & $\mathrm{Mo}$ & $\mathrm{Cu}$ \\
Weigth (\%) & 0.39 & 0.17 & 0.77 & 0.025 & 0.020 & 1.10 & 0.3 & 0.16 & 0.21 \\
\hline
\end{tabular}

Table 1: Chemical composition of the 42CrMo4 steel [26].

\begin{tabular}{lcc}
\hline Propriety & Abbreviation & Value \\
Yield's strength, $\mathrm{MPa}$ & $\sigma_{y}$ & 980 \\
Young's Modulus, GPa & $E$ & 206 \\
Ultimate tensile Strength, MPa & $\sigma_{u}$ & 1100 \\
Ultimate tensile deformation & $\varepsilon_{u}$ & 0.16 \\
Hardness & $\mathrm{HV}$ & 362 \\
\hline Cyclic yield strength, MPa & $\sigma^{\prime}{ }^{\prime}$ & 640 \\
Strain hardening exponent & $n^{\prime}$ & 0.12 \\
Fatigue strength coefficient, MPa & $\sigma_{f}^{\prime}$ & 1154 \\
Fatigue strength exponent & $\mathrm{b}$ & -0.061 \\
Fatigue ductility coefficient & $\boldsymbol{\varepsilon}_{f}^{\prime}$ & 0.18 \\
Fatigue ductility exponent & $\mathrm{c}$ & -0.529 \\
\hline
\end{tabular}

Table 2: Monotonic and cyclic properties of the 42CrMo4 steel [26].

\section{Specimens}

Several $42 \mathrm{CrMo} 4$ specimens were fabricated from $25 \mathrm{~mm}$ diameter rod. The raw material was cut and machined into several specimens, with the dimensions shown in Fig. 2.

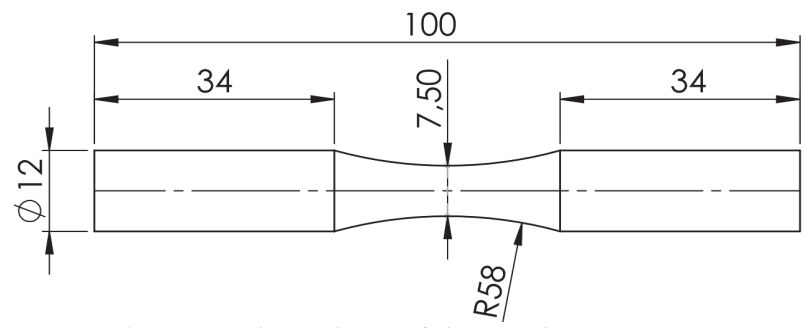

Figure 2: Dimensions of the specimens ( $\mathrm{mm})$. 


\section{Loading sequences}

To assess the performance of the SSF criterion with random loadings, two different loading paths where generated. These loading paths' plots, in the von Mises stress space, are illustrated in Fig. 3.

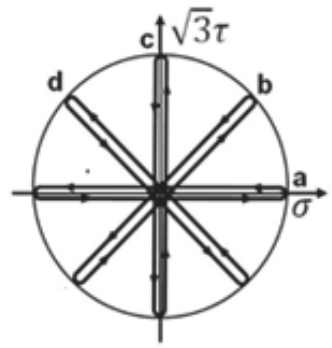

a)

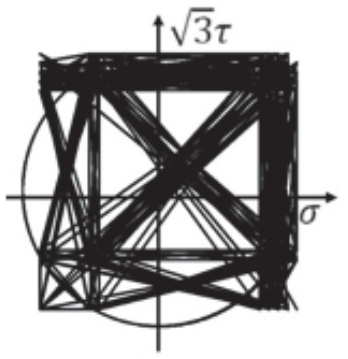

b)

Figure 3: Loading spectra with variable amplitude loading, (a) loading case ER, (b) loading case FSm.

The loading path ER (Fig. 3(a)) contains 4 branches, separated by $45^{\circ}$, and each branch is a full reversal. To make this loading path a random loading, the sequence in which each branch is applied must be random. Two random sequences, ER1 and ER2, were generated from this loading path. Both sequences were generated with the commercial software MATLAB [27] with the randi 0 function, resulting in two distinct loading sequences, each one with 100 branches, which are repeated until fracture. The number of times each branch occurs in a sequence is shown in Fig. 4. Each angle represents a different stress amplitude ratio.

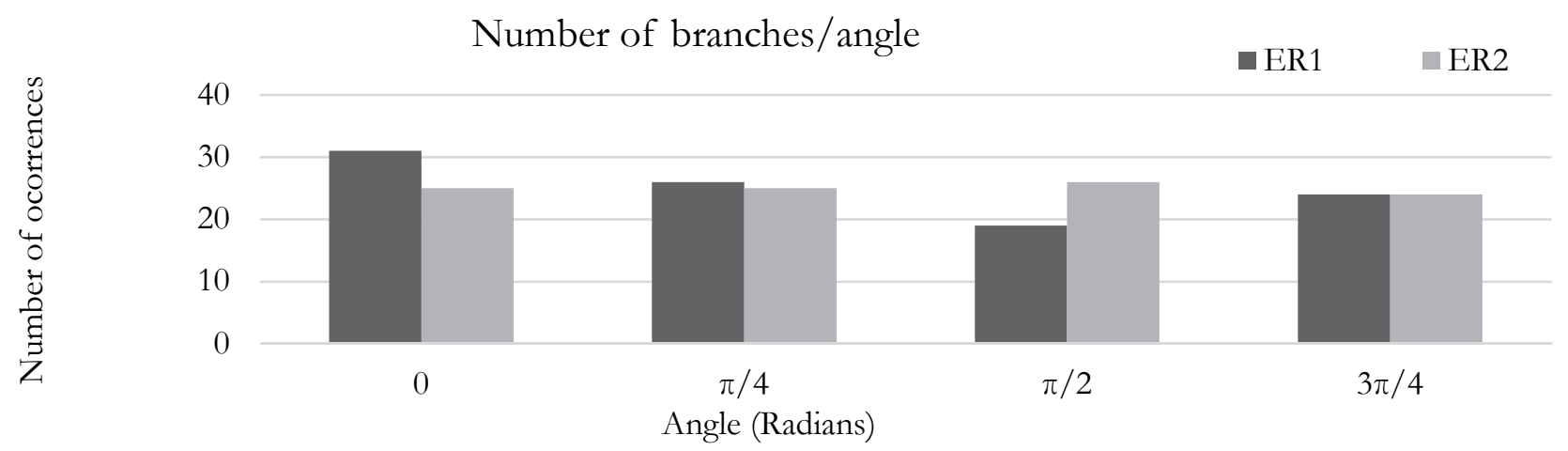

Figure 4: Number of branches per angle.

The non-random version of this loading path is designated as ENR. Fatigue data from random loadings is compared with the non-random results, retrieved from the work of Anes et al. [28].

Fig. 3(b) depicts the loading case FSm. This loading path is a variable amplitude loading that activates several loading phenomena such as proportionality and non-proportionality. The loading spectrum is a modified version of the well-known FALSTAFF loading spectrum, which has been obtained from the AFGROW Fracture Analysis Software [29]. FALSTAFF, which stands for Fighter Aircraft Loading Standard for Fatigue, released in 1975 [30], represents the load spectrum of the lower wing root panel from a combat aircraft, and contains data from about 200 flights with around 36,000 cycles. A portion of the spectrum was randomly selected, and then modified to fit the purpose of this work. From the retrieved portion of the original spectrum, all points between -0.4 and 0.4 are removed, and a factor of 1.2 is applied to all points, and those which result in an absolute value greater than 1.0 are removed. Lastly all points between 0.6 and 0.8 (in absolute value) are again increased by a factor of 1.2; this modification is needed to increase the damage in the specimen. The resulting spectrum, with 1500 data points, is applied to the axial component and, in reversed order, to the torsional component.

\section{Fractography analysis}

After failure, specimen's surfaces were inspected. With the aid of an electronic microscope, the initiation point of the fatigue crack was identified. Measured angles were compared with predictions from referenced critical plane models. 


\section{DAMAgE ACCUMULATION}

ab. 3 summarizes the experimental data for all loading cases. In the first column, it is specified the loading case. In the second column corresponds to que specimen designation. The third and fourth columns contain the maximum value of axial stress and shear stress, respectively, for each specimen. Fatigue life is given in blocks in the fifth column, where a block is defined as the spectrum history of the loading sequence.

\begin{tabular}{|c|c|c|c|c|}
\hline Case & Specimen designation & Axial $[\mathrm{MPa}]$ & Shear $[\mathrm{MPa}]$ & $\mathrm{Nf}$ \\
\hline \multirow{4}{*}{ ER1 } & ER1-501 & 501 & 290 & 1243 \\
\hline & ER1-523 & 523 & 303 & 748 \\
\hline & ER1-529 & 529 & 325 & 528 \\
\hline & ER1-546 & 546 & 323 & 626 \\
\hline \multirow{4}{*}{ ER2 } & ER2-498 & 498 & 288 & 1425 \\
\hline & ER2-505 & 505 & 292 & 2986 \\
\hline & ER2-521 & 521 & 301 & 1232 \\
\hline & ER2-530 & 530 & 307 & 706 \\
\hline \multirow{4}{*}{$\mathrm{ENR}^{*}$} & ENR-490 & 490 & 283 & 16458 \\
\hline & ENR-520 & 520 & 300 & 7823 \\
\hline & ENR-540 & 540 & 312 & 5525 \\
\hline & ENR-552 & 552 & 319 & 1040 \\
\hline \multirow{7}{*}{ FSm } & FSm-463 & 463 & 267 & 1955 \\
\hline & FSm-488 & 488 & 282 & 1085 \\
\hline & FSm-496 & 496 & 286 & 659 \\
\hline & FSm-517 & 517 & 299 & 542 \\
\hline & FSm-520 & 520 & 300 & 338 \\
\hline & FSm-541 & 541 & 312 & 283 \\
\hline & FSm-546 & 546 & 315 & 202 \\
\hline
\end{tabular}

Table 3: Experimental fatigue data for all loading cases. *Fatigue data retrieved from the work of Anes et al. [28].

Fig. 5 shows fatigue life correlation for all the loading cases considered. Fatigue life boundary factors of 2 and 3 are plotted with a dashed line and a solid line, respectively. Fatigue life was estimated in Fig. 5 with the full SSF package (damage criterion, block extraction method, cycle counting method of vcc and Miner's linear damage accumulation rule).

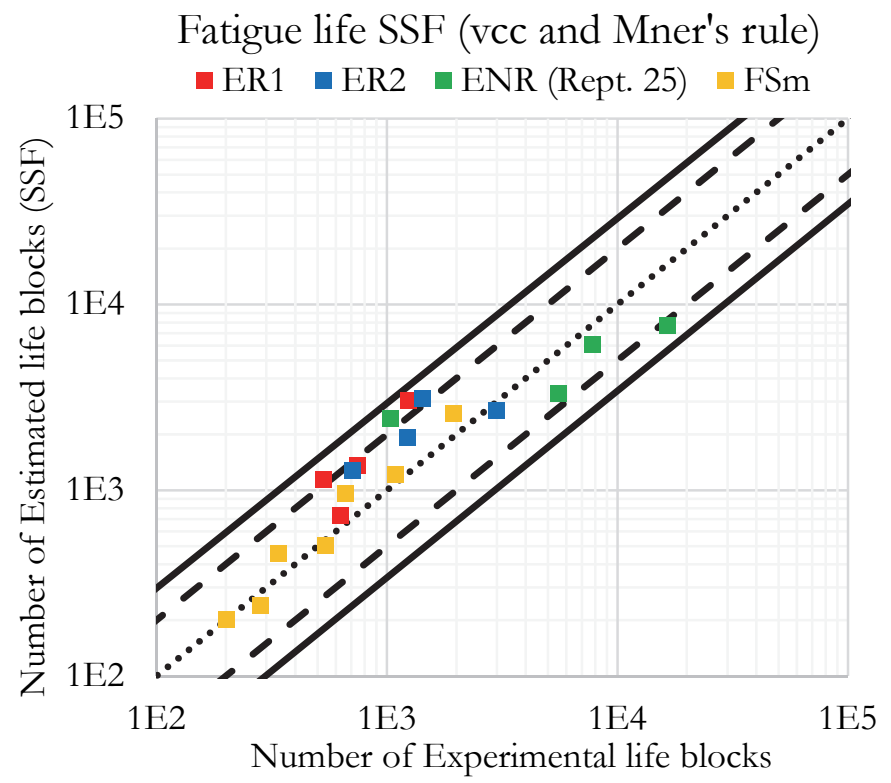

Figure 5: Fatigue life correlation with the SSF method applied with the vcc and Miner's rule (Experimental vs Estimated). 
In Fig. 5 each point corresponds to a specific stress level. All points are within the outer bounds and only 5 points are outside from the inner boundaries. It seems to be an underestimation behaviour of the SSF model that resulted in fatigue life estimates higher than the experimental ones, which is deducted from the higher density of points above the dotted line. In Fig. 6 the same SFF package was applied but with the Rainflow cycle counting method instead of the vcc method. The results show an underestimation within the 2 and 3 boundary factors, having only satisfactory results for the ER1 and ER2.

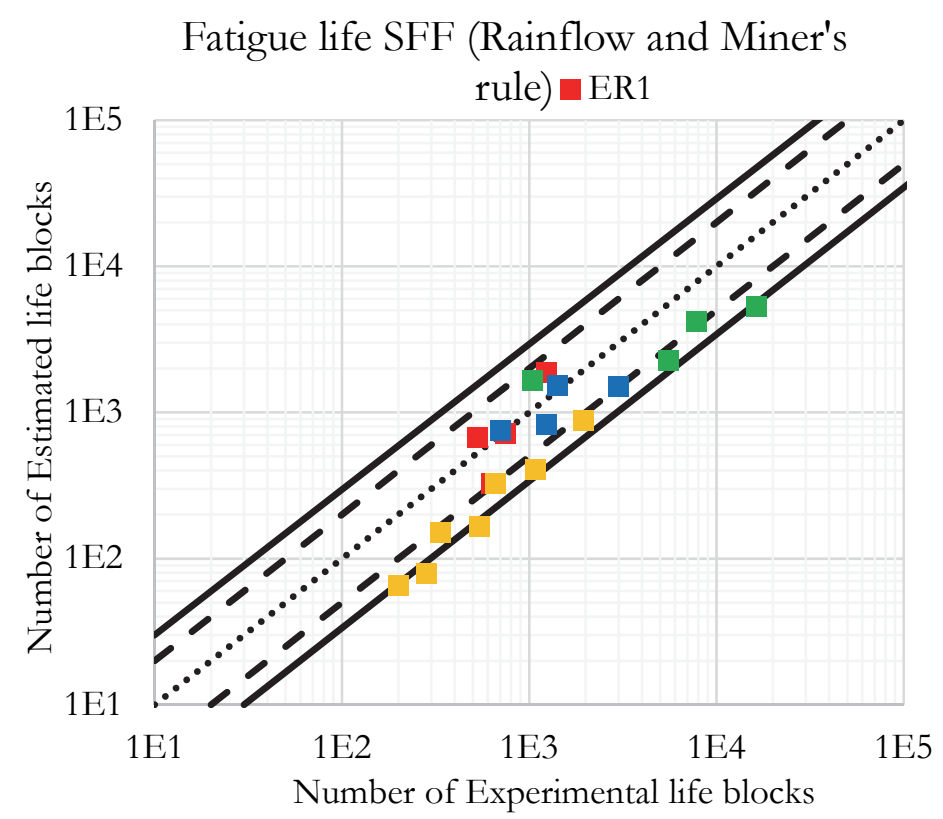

Figure 6: Fatigue life correlation with the SSF method applied with the vcc and Miner's rule (Experimental vs Estimated).

In Fig. 7 it is shown the fatigue life correlation, obtained for the W-B method. Fatigue life, which is presented in blocks, is calculated using Eq. (21).

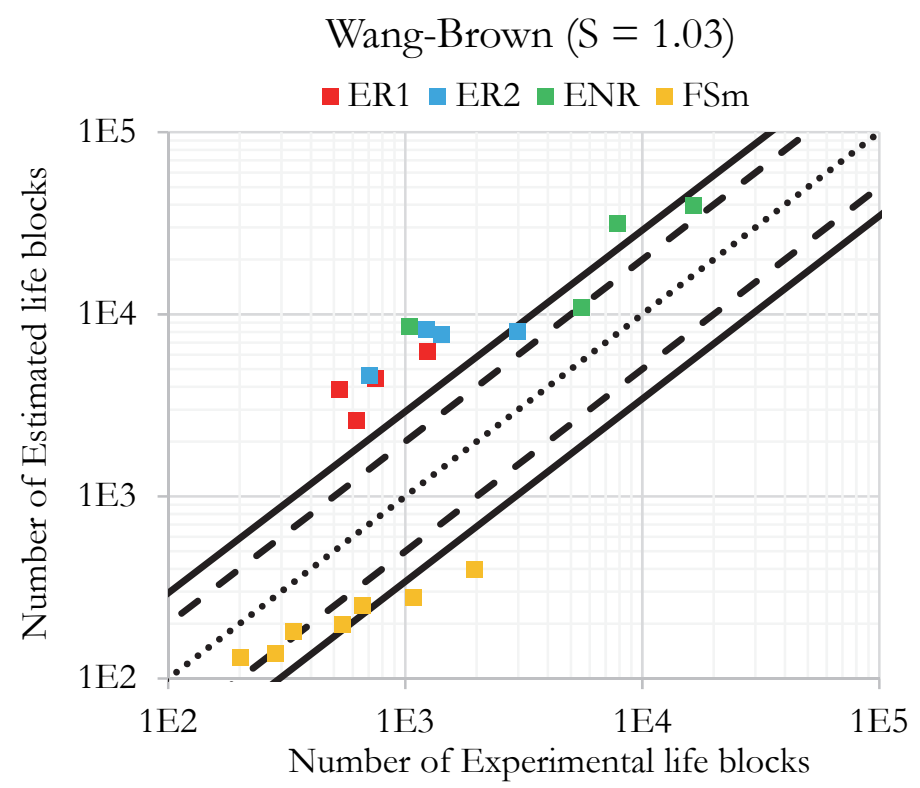

Figure 7: Fatigue life correlation with the W-B method.

The fatigue life estimates obtained with the W-B show a big scatter, where most of the estimations of the ER1 and ER2 loading sequences are above the upper life factor 3 boundary. This shows that the accumulated damage is lower than it 
should for these loadings. An opposite behaviour was shown with the FSm loading, where the accumulated damage was higher than it should be, leading to an underestimation of the fatigue life.

The damage accumulation approach was evaluated for W-B and SFF with both cycle counting methods vcc and Rainflow. Fig. 8 and Fig.9 present the accumulated damage, estimated at the time of fracture, using the W-B method and the SSF (vcc) method, where for the latter the Miner's and the Morrow's rules were applied. Fig 10 is also introduced in order to compare the estimated accumulated damage through the SFF Rainflow cycle counting method.

$$
\text { W-B vs SSF (vcc) }
$$



Figure 8: Accumulated damage at the time of fracture, for the ER1, ER2 and ENR loadings.

W-B vs SSF (vcc)

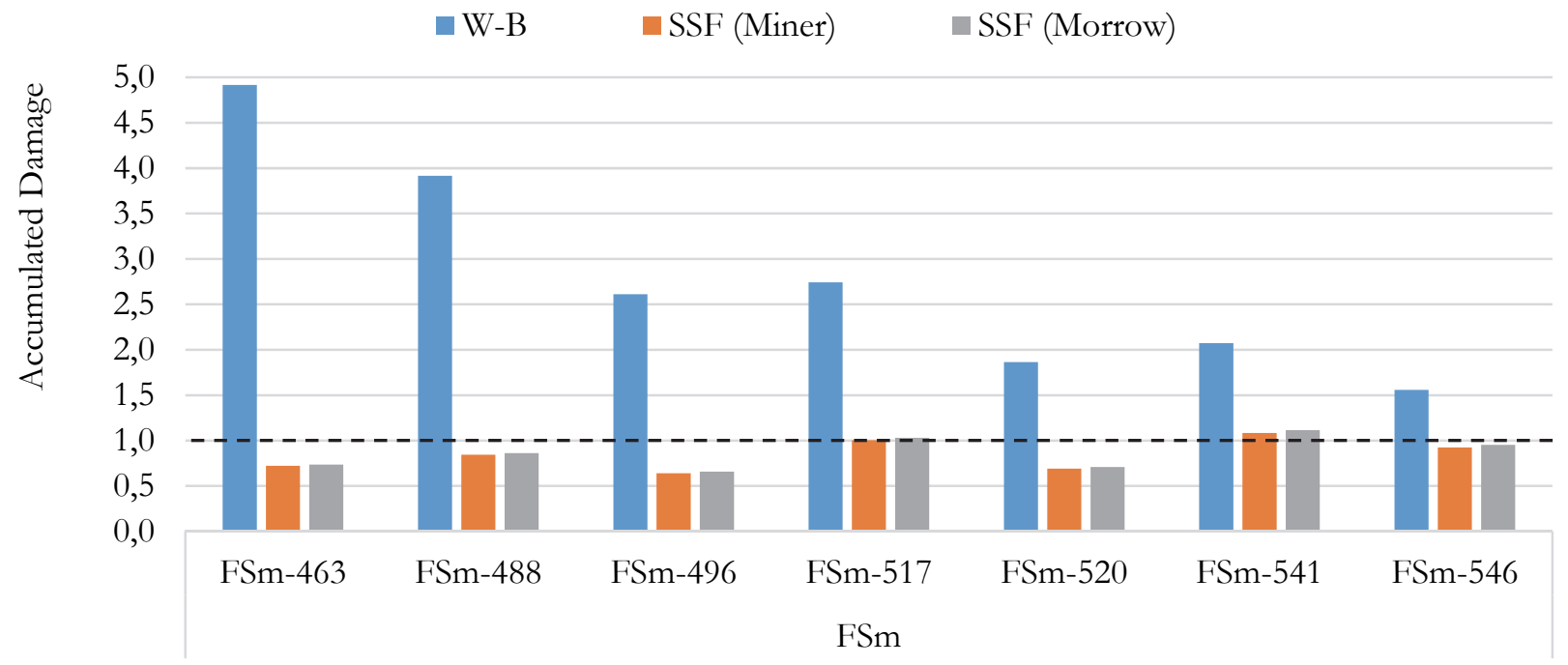

Figure 9: Accumulated damage at the time of fracture, for the FSm loading.

As seen in Fig. 8, the accumulated damage, for all the loadings and all stress levels, is too low with the W-B model. With SSF model the accumulated damage is much higher. However, in most of the stress levels of the ER1 and ER2 loadings the damage is almost half of what it should be. For the FSm loading estimates, shown in Fig. 9, the accumulated damage 
estimated with the W-B method was too high, as expected from the correlation shown previously in Fig. 7. However, the extra damage will lower as the stress level increases. The SSF results for the FSm loading case are much better, with most of the stress levels being near the unitary damage line. As seen in Fig. 8 and Fig. 9, there is not much difference between the use of the Morrow's rule and the Miner's rule. This is explained by the fact that the block extraction method only extracted a few blocks, due to the low variation of the SSF's eq. shear stress amplitude.

Through Fig. 10 it is observed that the estimation of accumulated damage with the Rainflow cycle counting showed very high values. This is due to the significant increase number of accounted cycles per bloc for all the applied loading sequences. The increase is more significant in the FSm loading sequence where the number of cycles withdrawn from the spectrum is three times higher than the considered by the vcc method. Only in ER1 and ER2 the results are satisfactory.



Figure 10: Accumulated damage at the time of fracture, for the four loading sequences

It is also relevant to compare the estimate crack plane and the measured in all the tested specimens for each of the four loading cases. One angle measurement and initiation point of fracture for each of the loading sequence obtained in the random loading sequences is presented in the Fig. 11.

With Tab. 4 a comparison is easily visible between the measured crack plane angles and the crack plane estimates, obtained with different critical plane models.

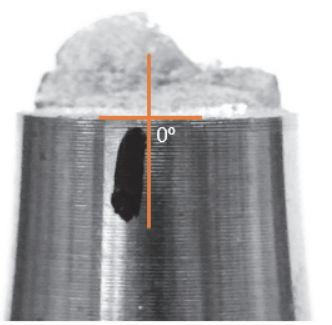

(A)
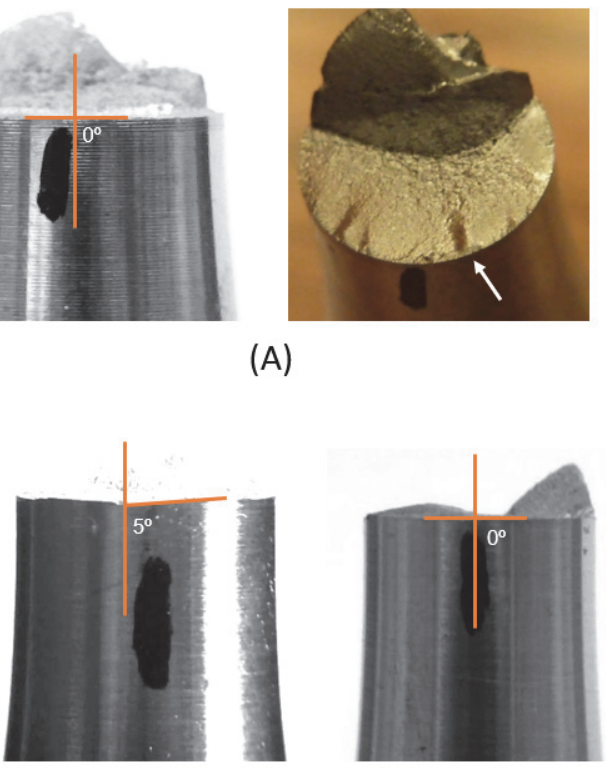
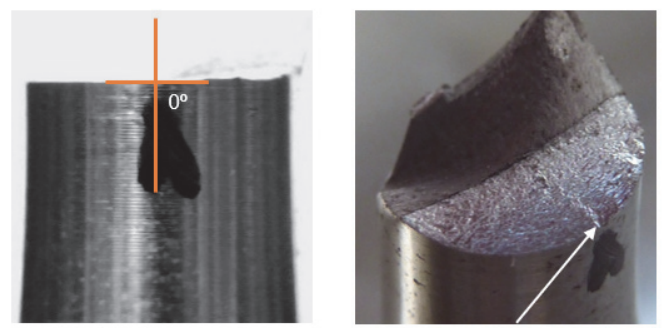

(B)
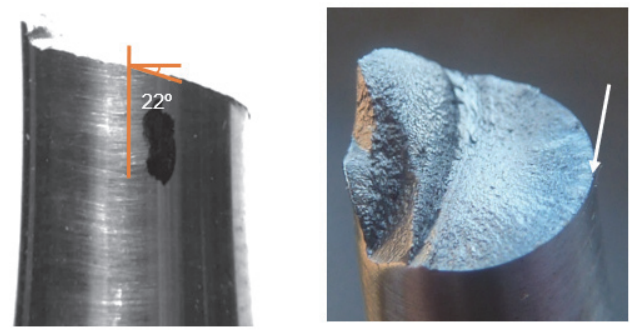

(C)

Figure 11: Example photographs of angles and initiation points of fracture obtained in (A) ER1 (B) FSm (C) ER2 


\begin{tabular}{ccccc}
\hline & ER1 & ER2 & ENR & FSm \\
Findley & $0^{\circ}$ & $0^{\circ}$ & $0^{\circ}$ & $21^{\circ}$ \\
B-M & $0^{\circ}$ & $0^{\circ}$ & $0^{\circ}$ & $21^{\circ}$ \\
S-W-T & $0^{\circ}$ & $0^{\circ}$ & $0^{\circ}$ & $24^{\circ}$ \\
F-S & $0^{\circ}$ & $0^{\circ}$ & $0^{\circ}$ & $20^{\circ}$ \\
LIU I & $0^{\circ}$ & $0^{\circ}$ & $0^{\circ}$ & $20^{\circ}$ \\
LIU II & $90^{\circ} / 0^{\circ} /+90^{\circ}$ & $90^{\circ} / 0^{\circ} /+90^{\circ}$ & $90^{\circ} / 0^{\circ} /+90^{\circ}$ & $-70^{\circ} /+20^{\circ}$ \\
Measured & $0^{\circ}$ & $-22^{\circ} / 0^{\circ} / 5^{\circ}$ & $0^{\circ}$ & $0^{\circ}$ \\
\hline
\end{tabular}

Table 4: Comparison of the measured crack plane angle with estimates.

For the ER1 and ENR loading cases, all models correctly predicted the crack plane for all stress levels. For the ER2 sequence, two of the specimens showed distinct crack plane angles. In all the criteria, in each plane, the damage parameter showed the same value for loadings ER1, ER2 and ENR, which share the same loading path. This behaviour shows that critical plane models do not consider the load sequence. For the FSm loading case, all the specimens fractured at $0^{\circ}$, and none of the selected criteria was able to predict that crack plane angle.

\section{CONCLUSIONS}

$\mathrm{I}$ $\mathrm{n}$ this work, one non-random and three random sequences were applied to specimens of the 42CrMo4 steel under different stress levels. Fatigue life from the ER1 and the ER2 loading sequences were considerably shorter than the lives obtained with the non-random equivalent loading sequence, ENR. The randomness introduced in the loading path, shared by these three loading sequences, significantly reduced fatigue life. For all the loading cases fatigue life was estimated by the SSF model using Miner and Morrows damage accumulation rule and using as cycle counting methods the Rainflow rule and vcc method. The difference between Miner and Morrow's rules is negligible in comparison with WangBrown in all loading cases. There is only a big difference between the SFF models when the cycle counting method is changed, having almost opposite behaviors, the Rainflow tends underestimate life and vcc tends to overestimate, but both are able to predict fatigue life under the boundary life factor 3 , which is commonly used in fatigue life correlation.

Damage accumulation, at time of fracture, was not only estimated with the SSF model but also with Wang-Brown's model (W-B). Wang-Brown's underestimated the damage of the ENR, ER1 and ER2 loading sequences, as oppose the damage overestimation shown in the FSm loading.

The SSF model also showed a tendency to underestimate damage, although, in a smaller scale, compared to W-B. Even though the SSF criterion, at its present state, can't account the mean stress effect, it showed good results when estimating fatigue life with non-proportional random loadings with variable stress amplitudes and mean stress, performing better than the W-B model. However, additional studies are needed to confirm, or not, the verified trend according to which the SSF equivalent stress tends to be lower than the value it should be under random loadings with variable amplitude.

\section{ACKNOWLEDGEMENTS}

This work was supported by FCT, through IDMEC, under LAETA, project UID/EMS/50022/2019.

\section{REFERENCES}

[1] Cui, W. (2002). A state-of-the-art review on fatigue life prediction methods for metal structures, Journal of Marine Science and Technology, 7(1), pp. 43-56. DOI: 10.1007/s007730200012.

[2] Campbell, G. S., Lahey, R. (1984). A survey of serious aircraft accidents involving fatigue fracture, International Journal of Fatigue, 6(1), pp. 25-30. DOI: 10.1016/0142-1123(84)90005-7.

[3] Gates, N., Fatemi, A. (2016). Multiaxial variable amplitude fatigue life analysis including notch effects, International Journal of Fatigue, 91(2), pp. 337-351. DOI: 10.1016/j.ijfatigue.2015.12.011.

[4] Freitas, M., Reis, L., Meggiolaro, M. A., de Castro, J.T.P. (2016). Comparison between SSF and Critical-Plane models to predict fatigue lives under multiaxial proportional load histories, Frattura ed Integrità Strutturale, 38, pp. 121-127.

DOI: 10.3221/IGF-ESIS.38.16. 
[5] Reis, L., Li, B., de Freitas, M. (2009). Crack initiation and growth path under multiaxial fatigue loading in structural steels, International Journal of Fatigue, 31, pp. 1660-1668. DOI: 10.1016/j.ijfatigue.2009.01.013.

[6] Papadopoulos, I. V. (2002). Critical plane approaches in high-cycle fatigue: on the definition of the amplitude and mean value of the shear stress acting on the critical plane, Fatigue \& Fracture of Engineering Materials \& Structures, 21(3), pp. 269-285. DOI: 10.1046/j.1460-2695.1998.00459.x.

[7] Manuel de Freitas, Luis Reis, and Bin Li (2006). Comparative study on biaxial low-cycle fatigue behaviour of three structural steels, Fatigue \& Fracture of Engineering Materials \& Structures, 29(12), pp. 992-999.

DOI: $10.1111 /$ j.1460-2695.2006.01061.x.

[8] Xia, T., Yao, W. (2013). Comparative research on the accumulative damage rules under multiaxial block loading spectrum for 2024-T4 aluminium alloy, International Journal of Fatigue, 48, pp. 257-265. DOI: $10.1016 /$ j.ijfatigue.2012.11.004.

[9] Palin-Luc, T., Lasserre, S. (1998). An energy based criterion for high cycle multiaxial fatigue, European Journal of Mechanics, 17(2), pp. 237-251. DOI: 10.1016/S0997-7538(98)80084-3.

[10] Morel, F., Morel, A., Nadot, Y. (2009). Comparison between defects and micro-notches in multiaxial fatigue - The size effect and the gradient effect, International Journal of Fatigue, 31(2), pp. 263-275. DOI: 10.1016/j.ijfatigue.2008.09.005.

[11] V. Anes, L. Reis, B. Li, M. Freitas, and C.M. Sonsino (2014). Minimum Circumscribed Ellipse (MCE) and Stress Scale Factor (SSF) criteria for multiaxial fatigue life assessment, Theoretical and Applied Fracture Mechanics, 73, pp. 109119. DOI: $10.1016 /$ j.tafmec.2014.08.008.

[12] Hertel, O., Vormwald, M. (2014). Multiaxial fatigue assessment based on a short crack growth concept, Theoretical and Applied Fracture Mechanics, 73, pp. 17-26. DOI: 10.1016/j.tafmec.2014.06.010.

[13] Louks, R., Gerin, B., Draper, J., Askes, H., Susmel, L. (2014). On the multiaxial fatigue assessment of complex threedimensional stress concentrators, International Journal of Fatigue, 63, pp. 12-24. Doi: 10.1016/j.ijfatigue.2014.01.001.

[14] Anes, V., Reis, L., Li, B., Fonte, M., de Freitas, M. (2014). New approach for analysis of complex multiaxial loading paths, International Journal of Fatigue, 62, pp. 21-33. DOI: 10.1016/j.ijfatigue.2013.05.004.

[15] Anes, V. (2015). Damage Analysis of Complex Loading Paths, Universidade de Lisboa, Instituto Superior Técnico, doctoral dissertation.

[16] Findley, W. N. (1958). A theory for the effect of mean stress on fatigue of metals under combined torsion and axial load or bending, Engineering Materials Research Laboratory, Division of Engineering, Brown University.

[17] Brown, M. W., Miller, K. J. (1973). A theory for fatigue failure under multiaxial stress-strain conditions, Proceedings of the Institute of Mechanical Engineers, 187(1), pp. 745-755. DOI: 10.1243/PIME_PROC_1973_187_069_02.

[18] Fatemi, A., Socie, D.F. (1988). A Critical Plane Approach to Multiaxial Fatigue Damage Including Out-of-Phase Loading, Fatigue and Fracture of Engineering Materials and Structures, 11(3), pp. 149-165.

DOI: $10.1111 / \mathrm{j} .1460-2695.1988 . t b 01169 . \mathrm{x}$

[19] Smith, K. N., Watson, P., Topper, T. H. (1970). A stress-strain function for the fatigue of metals, Journal of Materials, 5(4), pp. 767-778.

[20] Socie, D. F., Marquis, G. B. (2000). Multiaxial Fatigue. Society of Automotive Engineers, Warrendale, PA, EUA.

[21] KC Liu. (1993). A method based on virtual strain-energy parameters for multiaxial fatigue life prediction, ASTM special technical publication, 1191, pp. 67-67. DOI: 10.1520/STP24796S

[22] ASTM E1049, Standard Practices for Cycle Counting in Fatigue Analysis, ASTM International, 2011.

[23] Anes, V., Reis, L., Li, B., de Freitas, M. (2014). New cycle counting method for multiaxial fatigue, International Journal of Fatigue, 67, pp. 78-94. DOI: 10.1016/j.ijfatigue.2014.02.010

[24] Wang, C. H., Brown, M. W. (1996). Life prediction techniques for variable amplitude multiaxial fatigue - part 1: theories, ASME Journal of Engineering and Materials Technology, 118, pp. 367-370. DOI: 10.1115/1.2806821.

[25] Lv, Z., Huang, H-Z., Zhu, S-P., Gao, H., Zuo, F. (2015). A modified nonlinear fatigue damage accumulation model, International Journal of Damage Mechanics, 24, pp. 168-181. DOI: 10.1016/j.ijmecsci.2015.06.016.

[26] Reis, L. (2004). Comportamento Mecânico de Aços em Fadiga Multiaxial a Amplitude de Carga Constante e Síncrona, Universidade Técnica de Lisboa, Instituto Superior Técnico, doctoral dissertation.

[27] MATLAB Release 2015a, The MathWorks, Inc., Natick, Massachusetts, United States.

[28] Anes, V., Caxias, J., Freitas, M., Reis, L. (2017). Fatigue damage assessment under random and variable amplitude multiaxial loading conditions in structural steels, International Journal of Fatigue, 100, pp. 591-601.

DOI: $10.1016 /$ j.ijfatigue.2016.12.009

[29] AFGROW v5.2.5.19, LexTech, Inc., Centerville, Ohio, United States.

[30] Aircher, W., Branger, J., van Dijk, G. M., Ertelt, J., Hück, M., de Jonge, J. B. (1976). Description of a fighter aircraft loading for standard for fatigue evaluation. FALSTAFF, Common Report of FCW Emmen, LBF, NRL, IABG. 